PAPER • OPEN ACCESS

Induction heating system for die tooling of press for isothermal stamping of large-sized parts

To cite this article: V. Frizen et al 2020 IOP Conf. Ser.: Mater. Sci. Eng. 950012011

View the article online for updates and enhancements.

The Electrochemical Society

Advancing solid state \& electrochemical science \& technolog 2021 Virtual Education

Fundamentals of Electrochemistry:

Basic Theory and Kinetic Methods Instructed by: Dr. James Noël

Sun, Sept 19 \& Mon, Sept 20 at 12h-15h ET

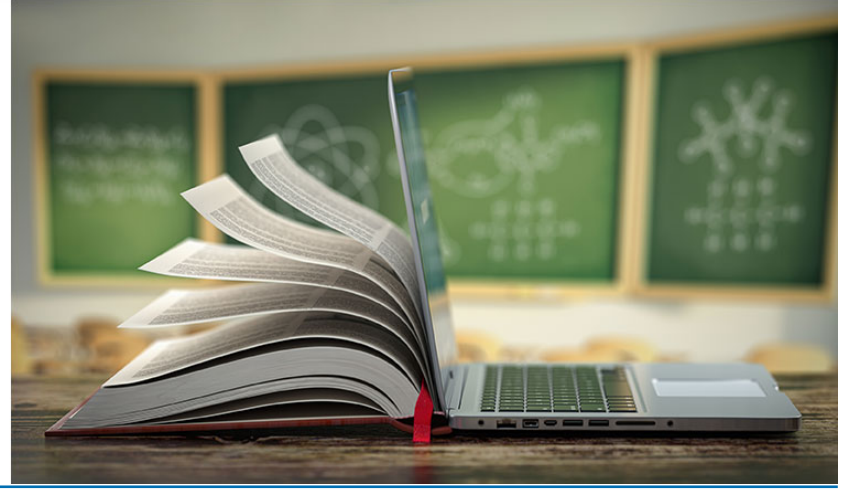




\title{
Induction heating system for die tooling of press for isothermal stamping of large-sized parts
}

\author{
V. Frizen, F Tarasov, S. Bychkov, S. Fatkullin, S. Sarapulov \\ Ural Federal University, Yekaterinburg, Russia \\ E-mail: F.E.Tarasov@urfu.ru
}

\begin{abstract}
.
The paper describes the installation for stamping large-sized parts. The use of induction heating for heating the working tool of the stamp in the operating mode is considered. A system with 4 flat inductors is described. This solution allows for the most efficient hot stamping operation compared to other known solutions. An example of the result of numerical simulation of the temperature field distribution is demonstrated.
\end{abstract}

\section{Introduction}

In the production of parts from wrought aluminum alloys by stamping, the costs of their machining are significantly reduced. Therefore, this method is in demand for mass production. To obtain a stamped part by this method, it is required to provide certain conditions that ensure the plasticity of the workpiece during pressing. In particular, the billet must be heated to a certain temperature when loaded into the press. This temperature must be maintained during the deformation of the billet in direct contact with the die tooling. The pressing of relatively small workpiece is fast enough. During pressing, the workpiece does not have time to transfer the accumulated heat to the die tooling. A certain amount of heat is released in the workpiece itself during the deformation process. Therefore, additional heating is not required to ensure the plasticity of the workpiece during pressing. However with an increase in the pressed part dimensions, the die tool dimensions, as well as the pressing time, grow. If you do not take additional steps to heat the die equipment in this case, the load on the die tool increases and the structure of the resulting part is disturbed. Therefore, in the production of large-sized stamped parts from aluminum alloys, preliminary heating of the stamping equipment is required.

The Fig. 1 shows a diagram of a die tool used for the production of large-sized parts from wrought aluminum alloys. The die and punch of the die is fixed to the parts of the hydraulic press using a series of traverses (intermediate steel plates). The area of these plates is gradually reduced to evenly distribute and transfer the force developed by the press directly to the die tool. These elements of the die equipment, as well as the tool itself, have a significant mass and are capable of accumulating a large amount of heat. The Fig. 2 shows a flow diagram of the production of a part using the above-described tool. The pressing section is completed with furnaces in which the stamped parts are heated, as well as the preheating of the stamping tool. At the end of a long heating process of the stamping tool, it is installed in a hot state on the press, after which the blanks are stamped. The stamping process can be continued as long as the surface of the stamping tool remains hot and is in direct contact with the blank being stamped.

cc (i) Content from this work may be used under the terms of the Creative Commons Attribution 3.0 licence. Any further distribution of this work must maintain attribution to the author(s) and the title of the work, journal citation and DOI. 


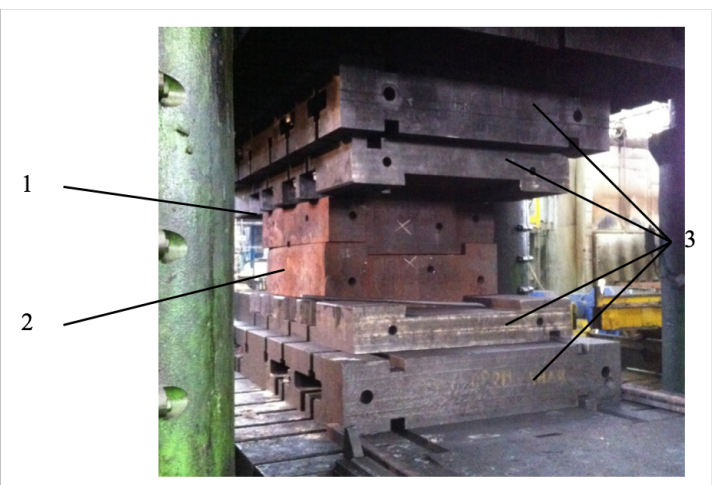

Figure 1. Die tooling design 1 - punch; 2 - matrix; 3 - traverses

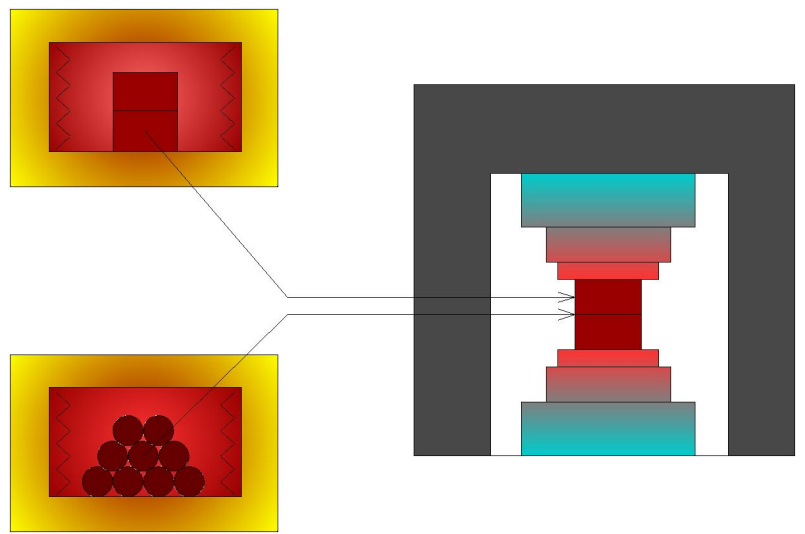

Figure 2. Technological scheme for preheating the stamping tool using 2 electric furnaces

As the tool surface temperature drops below the critical value, the die tool is removed from the press and loaded into the furnace for heating. Such a technological scheme is associated with significant labor costs for tool replacement. Since the die is mounted on cold die tooling plates, it loses heat at a high rate. Therefore, the tool replacement is necessary to make several times per day. To increase productivity and reduce energy consumption for the production of stamped blanks, tool heating is required directly during the pressing of parts. When compensating for the heat losses of a die tool, pressing of parts can be carried out without changing the tool during a work shift.

Previously, various schemes for heating the die tooling of presses were proposed (Fig. 3). For relatively small presses, variants of the design of the system for heating the outer surface of the die tool with gas burners or electric heaters $[1,2]$ were proposed. In this case, the die tooling is placed inside the heat-insulating chamber. This design is ineffective for large presses due to the insufficient specific surface power of the heaters to compensate for the heat loss of the preheated die tool for heating the traverse. In addition, stamping often requires the use of a lubricant that partially burns out when applied to the working surface of the stamp. The combustion products will inevitably settle on the inner surface of the heat-insulating chamber, which necessitates frequent stoppages of equipment for cleaning.

Since the main heat flux from the preheated die tool is directed towards the traverses, schemes for heating the tool with the location of the heat source directly in the traverses $[3,4]$ were 

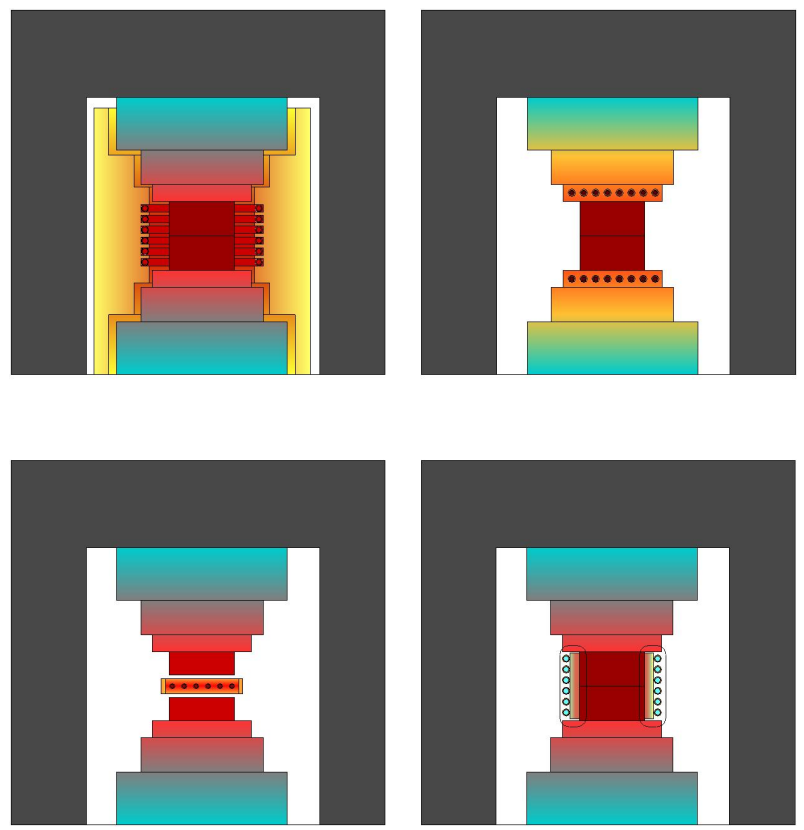

Figure 3. Variants of arrangement of die tooling heating systems

proposed. The possibility of placing heating elements in the traverses is limited by the conditions for ensuring the strength of the traverses, since these elements of the die equipment are under high mechanical stress. On the other hand, to accommodate heating elements of sufficient power, the number of cavities inside the traverse must be large. The use of electric heating elements in such heating systems for large die tooling did not justify itself due to insufficient efficiency in the presence of the above limitations.

Of particular interest are the systems for heating the working surface of the stamping tool $[5,6,7,8,9]$ in the intervals between stamping operations. The use in such heating systems as heat sources of electric heaters with a relatively low specific surface power is associated with an increase in the time between stamping cycles. This leads to a change in the rate in the technological production cycle. The maintenance of stamping equipment becomes more complicated. The use of induction heaters in this arrangement is practically impossible when changing tools, since the inductive wire must follow the inner surface of the die. It is also difficult to ensure the required uniformity of heating of the working surface during its induction heating.

In view of the above, the most promising in the production of large stamped parts from aluminum alloys is the induction heating system of the outer surface of the stamping tool. In this case, the surface of the stamp will be sufficient to accommodate the inductor of the required power, in addition, such a system is quite simple to control.

The inductor of the heating system can be a rectangular coil-solenoid made of a copper water-cooled tube, put on the corresponding part of the stamping tool [10]. The induction wire is insulated from the die tool. This design is the easiest to manufacture, but such an inductor is not universal. It is required to keep several inductors for different tools used in production. In addition, heating control in this case is only possible in one zone.

More multipurpose is the design of the inductor, made of flat coils attached through a layer of thermal insulation to each surface of the stamp separately (Fig. 4). In this case, each coil can be powered from a separate power source and the power can be controlled more flexibly on either side of the die. The problem with the versatility of the heating system is also removed. This inductor system can be placed on a large number of dies without changing the coil design. 


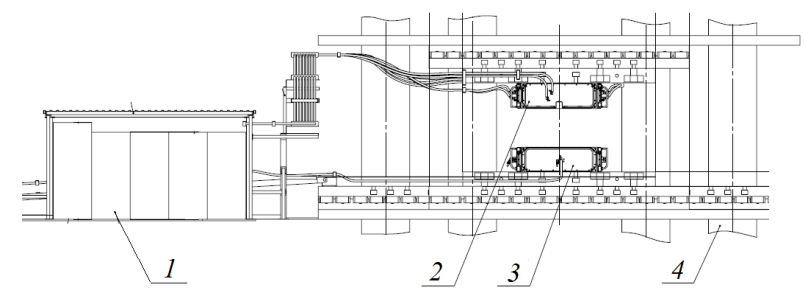

Figure 4. Induction heating system for die tooling with flat inductors 1 - power cabinet for the induction installation; 2 - a block of 4 inductors of the upper part of the tool; 3 - a block of 4 inductors of the lower part of the tool; 4 - press supports

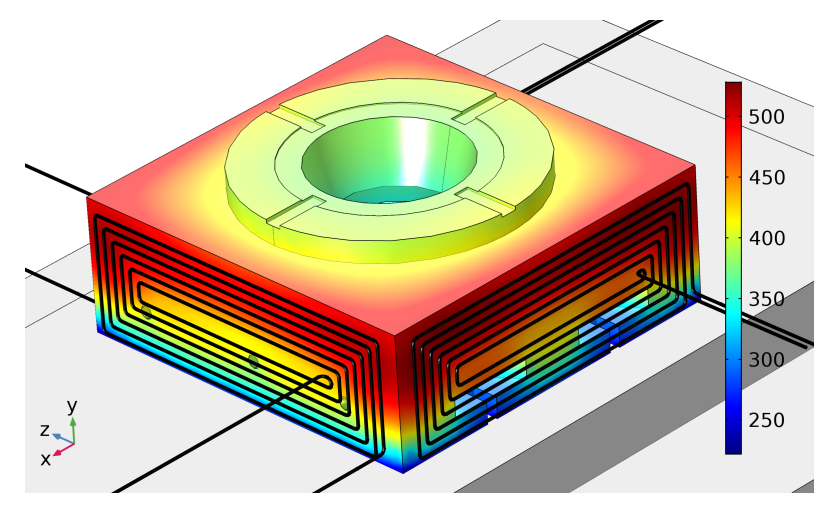

Figure 5. Temperature in a stamp working tool

Preliminary calculations have shown the high efficiency of the induction heating system for die tooling of the above-described design. The calculation results are shown in Fig. 5.

\section{Conclusions}

The review has shown the relevance and perspective of the development of systems for induction heating of die tooling for the production of large parts. In most cases, this heating method is the most acceptable both from the point of view of minimizing capital costs, and from the point of view of convenience and safety of operation, as well as maintenance of the press equipment.

\section{Acknowledgments}

This work was financially supported by the Ministry of Science and Higher Education of the Russian Federation, project No. 075-11-2019-028.

\section{References}

[1] Izakov I A 2005 Patent RU $2259901 C 1$

[2] Vasil'evich P N 2012 Patent RU 2464172 C1

[3] Bryan F E, Mitchell W D and Wheelon O A 1978 U.S. Patent US3783669A URL https://patents.google.com/patent/US3007427?oq=US3783669A

[4] Johns C L 2017 U.S. Patent US10267968B2 URL https://patents.google.com/patent/US10267968B2/en?oq=US2017066039

[5] Urban J DeSouza Robin M Forbes Jones B B H J A L L R S M S A S 2018 U.S. Patent US20190015890A1 URL https://patents.google.com/patent/US20190015890A1/en?oq=RU2550455

[6] Kunio Tomioka Masaaki Kita T $\mathrm{S} 1977$ U.S. Patent US4088000A URL https://patents.google.com/patent/US4088000A/en?oq=US4088000

[7] 2018 China Patent CN109909439A URL https://patents.google.com/patent/CN109909439A/en?oq=CN109909439

[8] 2017 China Patent CN207735540 URL https ://patents .google.com/patent/CN207735540U/en?oq=CN207735540 
IOP Conf. Series: Materials Science and Engineering 950 (2020) 012011 doi:10.1088/1757-899X/950/1/012011

[9] Shur I A 2013 Patent RU 2475329 C1

[10] Bychkov S A, Tarasov F E, Frizen V E and Smolyanov I A 2017 Stamp tool induction heating system 2017 18th International Conference on Computational Problems of Electrical Engineering (CPEE) pp 1-4 